\title{
Quantification of Left Atrium Fibrosis From LGE MRI in Atrial Fibrillation
}

\author{
Alice Andalò ${ }^{1}$, Claudio Fabbri ${ }^{1}$, Maddalena Valinoti ${ }^{1}$, Alessandro Masci ${ }^{1}$, Cristiana Corsi ${ }^{1}$ \\ ${ }^{1}$ University of Bologna, Cesena, Italy
}

\begin{abstract}
Late gadolinium enhanced magnetic resonance imaging (LGE-MRI) is a recent technique used for fibrosis distribution assessment in the left atrium (LA) wall. Unfortunately, LGE-MRI analysis does not rely on a standardized image processing protocol. The aim of the study was to compare different methods to quantify fibrosis in the LA in the $3 D$ domain. LGE-MRI from 60 $A F$ patients were analyzed applying five different approaches for fibrosis segmentation: an histogrambased (H-6SD), the image intensity ratio (IIR), the blood pool normalization $(B P)$, the Chan-Vese $(C V)$ and the graph-cut $(G C)$ method. For each method we quantified the percentage of fibrosis with respect to the entire LA wall mass. Results showed these five approaches can be divided in two groups; the group composed by the $\mathrm{H}$ $6 S D, C V$ and $G C$ is characterized similar results (mean coefficient of variation $=0.3$ ), while the results obtained applying BP and IIR strongly depend on the quality of the acquisition (mean coefficient of variation=0. 62). Utah stage classification resulted in a wide disagreement (22/60 patients, 37\%) among BP and IIR. The two approaches which best matched the Utah classification were the $C V$ and GC (49/60 patients, 82\%). This study confirmed the evaluation of the quantification method appears critical and further research is needed to define a satisfactory standard for atrial fibrosis segmentation.
\end{abstract}

\section{Introduction}

Atrial fibrillation (AF) is the most common sustained arrhythmia [1] and several studies have demonstrated that $\mathrm{AF}$ is associated with electrical and structural remodeling in the left atrium (LA) which was proven to sustain the arrhythmia [2,3].

Focusing on structural remodeling, late gadolinium enhanced magnetic resonance imaging (LGE-MRI) is a recent technique used for LA fibrosis localization and quantification in the LA wall. The DECAAF study [4] reported that extent of fibrosis in the LA in AF patients may predict recurrences after the ablation procedure. In [5] patients were divided into 4 categories depending on fibrosis extent: Utah stage 1 or minimal fibrosis $(<5 \%$ enhancement), Utah stage 2 or mild fibrosis (5-20\% enhancement), Utah stage 3 or moderate fibrosis (20-
$35 \%$ enhancement) and Utah stage 4 or extensive fibrosis (greater than 35\% enhancement). Based on this scoring different therapeutic approaches were suggested, including pulmonary vein isolation for patients in Utah score 1 and 2, pulmonary vein isolation and linear lesions in the LA posterior wall for patients in Utah score 3, ablation of the atrio-ventricular node and biventricular pacing for patients in Utah score 4. Therefore the information about the fibrosis extent may help the electrophysiologist in patient's selection for the ablation procedure.

Despite these promising results, assessment of fibrosis from LGE-MRI in AF patients has not been adopted in the clinical setting due to scarce result reproducibility $[6,7]$ mainly due to the absence of a standardized image processing protocol to quantify fibrosis extent. Therefore the aim of the study was to compare different methods to quantify fibrosis in the LA in the 3D domain.

\section{Materials and Methods}

\subsection{Clinical Data}

LGE-MRI from $60 \mathrm{AF}$ patients acquired at the CARMA Center (University of Utah) in which manual tracing of LA wall by expert radiologist was available, were analyzed [8].

All patients underwent MRI studies on a 1.5-T Avanto clinical scanner (Siemens Medical Solutions, Erlangen, Germany) using a TIM phased-array receiver coil or 32channel cardiac coil. LGE-MRI was acquired 15 minutes after the contrast agent injection (dose, $0.1 \mathrm{mmol} / \mathrm{kg}$ body weight; Multihance, Bracco Diagnostic Inc, Princeton, NJ) using 3-dimensional inversion-recovery-prepared, respiration-navigated, ECG gated, gradient-echo pulse sequence with fat saturation (voxel size of 1.251.252.5 $\mathrm{mm}$, flip angle of $22^{\circ}, \mathrm{TR} / \mathrm{TE}=6.1 / 2.4 \mathrm{~ms}, \mathrm{IT}=230$ to 320 $\mathrm{ms}$, parallel imaging with GRAPPA technique with 2 and 42 reference lines. Typical scan time for the DE-MRI study was 5 to 9 minutes, depending on the subject's respiration and heart rate.

\subsection{LGE-MRI Data Processing}

Five different approaches for fibrosis segmentation were applied in this study.

Thresholding techniques included a histogram-based 
reference, a blood-pool-based reference and the image intensity ratio technique [7]. All these approaches required a reference value Ref obtained from a region of interest (ROI):

$$
\mathrm{Th}=\operatorname{Ref}+\mathrm{N} \cdot \mathrm{SD}
$$

where Th is the threshold that separates scar tissue from healthy tissue, SD is the standard deviation in the selected ROI, $\mathrm{N}$ is the number of SDs.

In the histogram-based reference approach (H-6SD), normal tissue is defined as the mean of the pixel intensities between 2 and $40 \%$ of the maximum intensity within the atrial wall $\left(\mathrm{M}_{2-40 \%}\right)$. The threshold $\mathrm{Th}_{\mathrm{H}-6 \mathrm{SD}}$ was computed at $6 \mathrm{SD}\left(\mathrm{SD}_{2-40 \%}\right)$ above the mean of normal tissue:

$$
\mathrm{Th}_{\mathrm{H}-6 \mathrm{SD}}=\mathrm{M}_{2-40 \%}+6 \mathrm{SD}_{2-40 \%}
$$

In other approaches, the ROI is located in the blood pool (BP) [9]. In this case the threshold was computed using the mean $\left(\mathrm{M}_{\mathrm{BP}}\right)$ and standard deviation $\left(\mathrm{SD}_{\mathrm{BP}}\right)$ of the pixel intensities inside the blood pool region:

$$
\mathrm{Th}_{\mathrm{BP}}=\left(\mathrm{I}_{\mathrm{LA}}-\mathrm{M}_{\mathrm{BP}}\right) / \mathrm{SD}_{\mathrm{BP}}
$$

where $\mathrm{I}_{\mathrm{LA}}$ is the myocardial intensity of the LGE-MRI in the LA wall.

In the image intensity ratio approach (IIR), the threshold is computed by normalizing the myocardial image intensity by the mean blood-pool intensity:

$$
\mathrm{Th}_{\mathrm{IIR}}=\mathrm{I}_{\mathrm{LA}} / \mathrm{M}_{\mathrm{BP}}
$$

Two additional techniques were based on the ChanVese (CV) and on a graph-cut (GC) models.

The CV approach [10] finds in the image the two regions characterized by the maximum difference in gray scale by minimizing the following energy function $F$ :

$$
F(C)=\int_{\operatorname{in}(C)}\left|I(x, y)-c_{1}\right|^{2} d x d y+\int_{\operatorname{out}(C)}\left|I(x, y)-c_{2}\right|^{2} d x d y
$$

where $I$ is the image and $c$ are the mean intensities inside and outside the evolving curve $C$.

In the graph based approach [11] the source and sink nodes were modeled as healthy and fibrotic tissue voxels. The energy function to be minimized was defined considering a regional term $R_{x}$ and a boundary term $B_{x, y}$ equally weighted $(\lambda=0.5)$ where $X$ are the nodes and $A$ the relationships between two adjacent voxels:

$$
F(L)=\lambda \sum_{x \in X} R_{x}\left(L_{x}\right)+(1-\lambda) \sum_{(x, y) \epsilon A} B_{x, y}\left(L_{x}, L_{y}\right)
$$
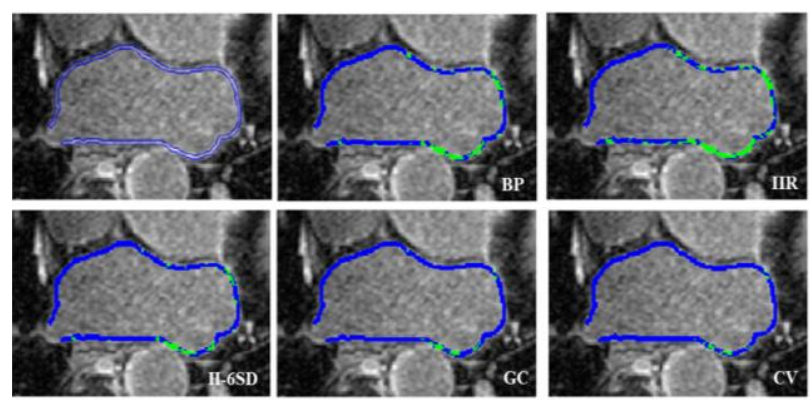

Fig. 1: A qualitative example of the fibrotic tissue (green region) evaluated in the LA wall (blue region) in one LGEMRI slice applying the different quantification techniques (BP: blood-pool-based reference; IIR: image intensity ratio; $\mathrm{CV}$ : Chan-Vese; GC: graph-cut; H-6SD: histogram-based reference).

The LA wall was modeled using two Gaussian functions representing the two classes, healthy myocardium $\left(\mu_{1}, \sigma_{1}\right)$ and fibrotic tissue $\left(\mu_{2}, \sigma_{2}\right)$. The regional term was defined as:

$$
\begin{aligned}
& R_{x}(\text { healthy })=-\ln P\left(\text { healthy } \mid I_{x}\right)=-\ln \left(\frac{\pi_{1} N\left(I_{x} \mid \mu_{1}, \sigma_{1}\right)}{p\left(I_{x}\right)}\right) \\
& R_{x}(\text { fibrosis })=-\ln P\left(\text { fibrosis } \mid I_{x}\right)=-\ln \left(\frac{\pi_{2} N\left(I_{x} \mid \mu_{2}, \sigma_{2}\right)}{p\left(I_{x}\right)}\right)
\end{aligned}
$$

where $I_{x}$ is the intensity of the voxel $x, \pi_{k}$ represents the ratio of the two classes, $\mu_{k}$ are the mean and $\sigma_{k}$ the covariance matrix of the two Gaussian models; $N(\cdot)$ describes the probability Gaussian function and $p\left(I_{x}\right)$ is

$$
p\left(I_{x}\right)=\sum_{k=1}^{2} \pi_{k} N\left(I_{x} \mid \mu_{k}, \sigma_{k}\right)
$$

The boundary term was defined as:

$$
B_{x, y}\left(I_{x}, I_{y}\right)=\frac{e^{-\beta\left\|I_{x}-I_{y}\right\|^{2}}}{\operatorname{dist}(x, y)}
$$

where $I_{x}$ and $I_{y}$ are the intensity of the voxel $x$ and of its neighbor $y ; \beta$ is a penalty term which was set equal to 5 to give more weight to high gradients between voxels belonging to different classes; $\operatorname{dist}(x, y)$ is the Euclidean distance between the two voxels $x$ and $y$. Minimizing the energy function $F(L)$ leads to the partition of the graph in two regions corresponding to healthy and fibrotic tissue.

All LGE-MRI slices were analyzed for each patient by considering the LA wall manually traced by the expert cardiologist.

For each patient and for each technique we obtained the 3D model of the LA with visual localization of fibrosis. For each technique we quantified the percentage 

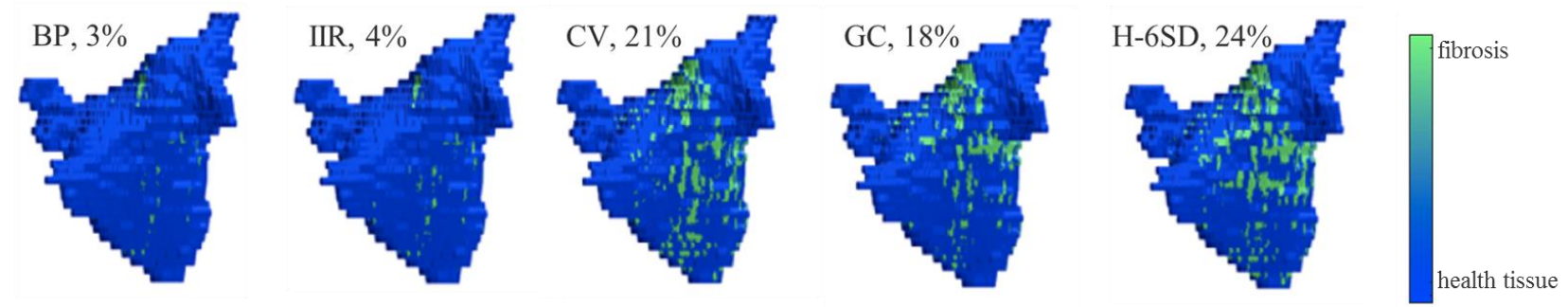

Fig. 2: Example of the LA 3D models obtained in one patient applying the five different approaches and the corresponding percentage of fibrotic tissue (BP: blood-pool-based reference; IIR: image intensity ratio; CV: Chan-Vese; GC: graph-cut; H-6SD: histogram-based reference).

of fibrosis with respect to the entire LA wall mass.

\section{Results}

A qualitative example of the fibrotic tissue evaluated in the LA wall in one LGE-MRI slice applying the different quantification techniques is reported in Figure 1.

An additional example of the LA 3D models obtained in one patient applying the five different approaches and the corresponding percentage of fibrotic tissue are reported in Figure 2.

Overall, results showed these five approaches can be divided in two groups; the group composed by the $\mathrm{H}$ $6 \mathrm{SD}, \mathrm{CV}$ and $\mathrm{GC}$ is characterized by similar results (mean coefficient of variation=0.3), while the results obtained applying BP and IIR strongly depend on the quality of the acquisition (mean coefficient of variation $=0.62$ ).

Considering H-6SD technique as the reference approach, BP and IIR showed a negative and low correlation; GC and CV showed a positive and good correlation (Figure 3).

An example of Utah stage score obtained in one patient applying the different techniques for fibrosis segmentation is shown in Table 1. Utah stage classification resulted in a wide disagreement (22/60 patients, 37\%) among BP and IIR. The two approaches which best matched the Utah classification were the CV and GC (49/60 patients, $82 \%)$

\section{Discussion and Conclusion}

This study confirmed the evaluation of the quantification method appears critical and further research is needed to define a satisfactory standard for the segmentation of atrial fibrosis.

BP and IIR use the blood pool region to normalize the intensity values on the LA wall; therefore these techniques strongly depend on image quality. The LA cavity signal should be properly suppressed but unfortunately a standardized image acquisition protocol is not available. Its availability would improve the performance of methods based on blood pool reference
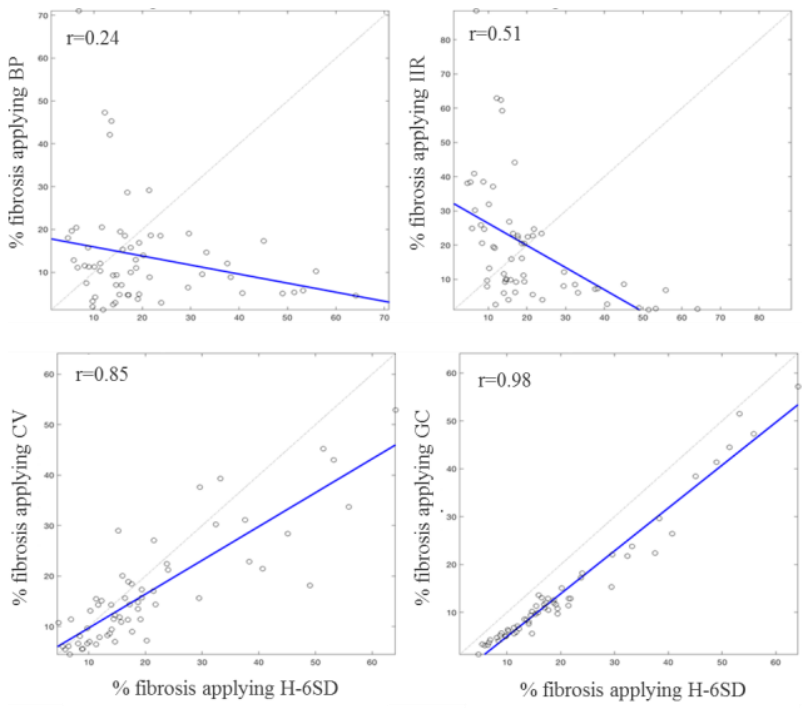

Fig. 3: Correlation analysis between $\%$ of fibrotic tissue obtained applying the different techniques. The H-6-sd was considered the reference technique (BP: blood-pool-based reference; IIR: image intensity ratio; CV: Chan-Vese; GC: graph-cut; H-6SD: histogram-based reference; p: correlation coefficient).

Table 1. Percentage of fibrosis obtained applying the five segmentation approaches and the derived Utah score and therapeutic indication in Patient \#0937.

\begin{tabular}{|c|c|c|c|}
\hline & $\%$ of fibrosis & Utah stage & therapeutic indication \\
\hline $\mathbf{H}_{6-\mathrm{SD}}$ & $53,2 \%$ & IV & $\begin{array}{l}\text { ablation of AV node and } \\
\text { biventricular pacing }\end{array}$ \\
\hline BP & $5,7 \%$ & I & pulmonary vein isolation \\
\hline IIR & $1,5 \%$ & I & pulmonary vein isolation \\
\hline $\mathrm{CV}$ & $43,0 \%$ & IV & $\begin{array}{l}\text { ablation of AV node and } \\
\text { biventricular pacing }\end{array}$ \\
\hline GC & $51,5 \%$ & IV & $\begin{array}{l}\text { ablation of AV node and } \\
\text { biventricular pacing }\end{array}$ \\
\hline
\end{tabular}


values. H-6SD, CV and GC showed repeatable results.

In conclusion the choice of the approach to quantify fibrosis appears critical. Further research is needed to define a satisfactory standard in the segmentation of atrial fibrosis. In addition, there is a strong need for a standardized imaging protocol, especially for methods that exploit information from the blood-pool region.

\section{References}

[1] C.A. Morillo, A. Banerjee, P. Perel, D. Wood, and X. Jouven, Atrial fibrillation: the current epidemic. J Geriatr Cardiol 2017;14(3):195-203.

[2] M.A.J. Allessie, U. Schotten, Electrical, contractile and structural remodeling during atrial fibrillation. Cardiovascular Research 2002;54:230-236.

[3] G.B. Casaclang-Verzosa, T.S. Tsang, Structural and functional remodeling of the left atrium: Clinical and therapeutic implications for atrial fibrillation. Journal of the American College of Cardiology 2008;51:1-11.

[4] N.F. Marrouche, D. Wilber, G. Hindricks, P. Jais, Association of Atrial Tissue Fibrosis Identified by Delayed Enhancement MRI and Atrial Fibrillation Catheter Ablation: The DECAAF Study. JAMA 2014;311(5):498506.

[5] N. Akoum, M. Daccarett, C. McGann, N. Segerson, G. Vergara, S. Kuppahally, T. Badger, N. Burgon, T. Haslam, E. Kholmovski et al., Atrial Fibrosis Helps Select the Appropriate Patient and Strategy in Catheter Ablation of Atrial Fibrillation: A DE-MRI Guided Approach. Journal of

[6] R. Karim, R.J. Housden, M. Balasubramaniam, Z. Chen, D.1 Perry, A. Uddin, Y. Al-Beyatti, E. Palkhi, P. Acheampong, S. Obom et al., Evaluation of current algorithms for segmentation of scar tissue from late Gadolinium enhancement cardiovascular magnetic resonance of the left atrium: An open-access grand challenge. Journal of Cardiovascular Magnetic Resonance 2013;15:105.
[7] G. Pontecorboli, R.M. Figueras i Ventura, A. Carlosena, E. Benito, S. Prat-Gonzales, L. Padeletti, L. Mont. Use of delayed-enhancement magnetic resonance imaging for fibrosis detection in the atria: A review. Europace 2016;19:euw053.

[8] R.S. Oakes, T. Badger, E. Kholmovski, N. Akoum, N.S. Burgon, E.N. Fish, J. Blauer, S. Rao, E. Dibella, N.M. Segerson et al., Detection and Quantification of Left Atrial Structural Remodeling With Delayed-Enhancement Magnetic Resonance Imaging in Patients With Atrial Fibrillation. Circulation 2009;119:1758-67.

[9] L. Malcolme-Lawes, C. Juli, R. Karim, W. Bai, R. Quest, P. Boon Lim, S. Jamil-Copley, P. Kojodjojo, B. Ariff, D.W. Davies et al., Automated analysis of atrial late gadolinium enhancement imaging that correlates with endocardial voltage and clinical outcomes: A 2-center study. Heart Rhythm: the official journal of the Heart Rhythm 2013; 10:1184-91.

[10] T.F. Chan, L.A. Vese, Active contours without edges. IEEE Transactions on Image Processing 2001;10(2):266-277.

[11] Y. Boykov, V. Kolmogorov, An Experimental Comparison of In-Cut/Max-Flow Algorithms for Energy Minimization in Vision. IEEE Transactions on Pattern Analysis and Machine Intelligence 2004;26:1124-37.

Address for correspondence.

Alice Andalò

DEI, University of Bologna

Via Risorgimento 2, 40136 Bologna, Italy

E-mail address: alice.andalo@studio.unibo.it 